\title{
ATIVIDADES EDUCATIVAS PARA PORTADORES DE DOENÇA CRÔNICA: SUBSÍDIOS PARA A ENFERMAGEM
}

\author{
Elis Martins ULBRICH ${ }^{\mathrm{a}}$, Mariluci Alves MAFTUM ${ }^{\mathrm{b}}$, \\ Liliana Maria LABRONICI ${ }^{c}$, Maria de Fátima MANTOVANI ${ }^{\mathrm{d}}$
}

\section{RESUMO}

Trata-se de pesquisa de intervenção, realizada em uma Unidade Básica de Saúde do município de Colombo, Paraná, de março a novembro de 2009, com 35 portadores de doença crônica, com idades entre 18 e 60 anos, cadastrados no Programa de Hipertensão e Diabetes. Os objetivos foram: identificar o conhecimento sobre a hipertensão arterial e intervir mediante atividades educativas em grupo. A coleta de dados ocorreu por meio de entrevista semiestruturada e quatro encontros em grupos, e da sua análise emergiram as categorias: "Compreensão da doença" e "Maneiras de cuidar". Constatou-se que os usuários conhecem a doença, os seus fatores de risco e as possíveis complicações, e que as atividades educativas favoreceram a troca de experiências, proporcionaram a reflexão e a possibilidade de gestão de seu tratamento, e é uma estratégia que deve ser utilizada e divulgada pelos enfermeiros.

Descritores: Doença crônica. Educação em saúde. Enfermagem.

\section{RESUMEN}

Esta es una investigación de intervención, realizada en una Unidad Básica de Salud en la ciudad de Colombo, Paraná, de marzo a noviembre de 2009, con 35 pacientes con enfermedades crónicas de entre 18 y 60 años, inscritos en el Programa de Hipertensión y Diabetes. Los objetivos fueron: determinar los conocimientos sobre la hipertensión, e intervenir a través de actividades educativas de grupo. La recolección de datos fue a través de entrevistas semiestructuradas y cuatro reuniones de grupo, y de su análisis surgieron las categorías: "La comprensión de la enfermedad" y "Maneras de cuidar". Se encontró que los usuarios conocen la enfermedad, sus factores de riesgo y las posibles complicaciones y que las actividades educativas facilitaron el intercambio de experiencias, proporcionaron la reflexión y la posibilidad de gestionar su tratamiento, y es una estrategia que debe ser utilizada y propagada por los enfermeros.

Descriptores: Enfermedad crónica. Educación en salud. Enfermería.

Título: Actividades educativas para personas con enfermedades crónicas: subsidios para la enfermería.

\section{ABSTRACT}

This is an intervention study conducted in a Unidade Básica de Saúde (Basic Health Unit) in Colombo, Parana, Brazil, from March to November 2009, with 35 carriers of cronic diseases aged between 18 and 60 years, and enrolled in the Hypertension and Diabetes Program. The objectives were to identify their knowledge about arterial hypertension and act through educational group activities. Data were collected though semistructured interviews and four group meetings, and the following categories emerged from the analysis: "Understanding of the disease" and "Ways of caring". It was found that users knew the disease, its risk factors and possible complications, and that educational activities favored the sharing of experiences, provided reflection and the possibility of treatment management. This is a strategy that should be used and promoted by nurses.

Descriptors: Chronic disease. Health education. Nursing.

Titles: Educational activities for people with chronic disease: grants for nursing.

a Doutoranda em Enfermagem da Universidade Federal do Paraná(UFPR). Bolsista REUNI. Membro do Grupo de Estudos Multiprofissional em Saúde do Adulto (GEMSA). Curitiba-PR, Brasil

b Doutora em Enfermagem. Professora Adjunta do Departamento de Enfermagem da UFPR. Vice-lider do Núcleo de Estudos, Pesquisa e Extensão em Cuidado Humano e de Enfermagem (NEPECHE). Curitiba-PR, Brasil.

c Doutora em Enfermagem. Professora Associada do Departamento de Enfermagem da UFPR. Líder do GEMSA. Curitiba-PR, Brasil

d Doutora em Enfermagem. Professora Associada do Departamento de Enfermagem da UFPR. Membro do GEMSA. Curitiba-PR, Brasil. 


\section{INTRODUÇÃO}

As doenças crônicas podem ser entendidas como uma série de agravos que pelo seu curso prolongado e sua multiplicidade de fatores de risco, requerem monitoramento constante e tratamento permanente. Dentre as doenças crônicas não transmissíveis encontramos as cerebrovasculares, cardiovasculares, renovasculares, respiratórias, neoplasias e diabetes mellitus que pertencem a essa classificação ${ }^{(1)}$.

Nos países em desenvolvimento as doenças crônicas são consideradas um problema de saúde pública, em função de possuírem alta prevalência, mortalidade, dificuldade de aceitação e adesão por seus portadores. Geralmente estas doenças aparecem no nível de atenção primária, e é neste que seu tratamento deveria ser realizado, no entanto, grande parte da atenção básica está voltada para o atendimento de problemas agudos e às necessidades mais urgentes dos usuários ${ }^{(1)}$.

Em 2005, cerca de 35 milhões de pessoas no mundo morreram de doenças crônicas, o que corresponde ao dobro das mortes relacionadas às doenças infecciosas ${ }^{(2)}$. No universo das doenças crônicas a Hipertensão Arterial Sistêmica (HAS) está entre a de maior ocorrência, e é considerada um dos principais fatores de risco para o desenvolvimento de enfermidades cardiovasculares, acidente vascular cerebral (AVC), infarto agudo do miocárdio (IAM) e afecções renais ${ }^{(3)}$.

No Brasil, o número de portadores de hipertensão arterial cresce a cada ano, e seu aparecimento é precoce. Estima-se que 4\% das crianças e adolescentes sejam portadores desta doença, e seu total no país é de aproximadamente 17 milhões de pessoas ${ }^{(3)}$. Em 2006, 28,9\% dos óbitos foram decorrentes de doenças cardiovasculares ${ }^{(4)}$, atingindo em 2003, $37 \%$ dos óbitos quando excluídos os decorrentes de causas mal definidas e/ou ainda por conseqüência da violência. Entre os fatores de risco para mortalidade, a hipertensão arterial explica $40 \%$ das mortes por acidente vascular cerebral e $25 \%$ daquelas por doença coronariana ${ }^{(5)}$.

Embora as causas da Hipertensão Arterial Sistêmica sejam desconhecidas, vários são os fatores que podem estar associados ao aumento dos níveis tensionais, como: o sedentarismo, o estresse, o tabagismo, o envelhecimento, antecedentes familiares, o gênero, o peso e os hábitos de vida ${ }^{(5)}$. Todavia, é possível reduzir mais de $50 \%$ dos óbitos decorrentes da HAS, se houver estratégias para enfrentar os principais fatores de risco $^{(2)}$, pois os portadores de hipertensão enfrentam mudanças no estilo de vida, e têm de incorporar a idéia de conviver com a doença, que para ser controlada, precisa da adesão ao tratamento medicamentoso e não medicamentoso, a fim de minimizar as complicações. Além disso, é indispensável acompanhar as co-morbidades como o diabetes, a dislipidemia e a obesidade ${ }^{(5)}$.

A aderência à terapêutica envolve um consenso entre profissional e o cliente acerca da forma mais apropriada de conduzir seu regime terapêutico. Os principais fatores envolvidos à adesão ao tratamento estão relacionados ao conhecimento, à percepção da hipertensão arterial, a motivação pessoal pela busca de melhor estado de saúde, e a obtenção do controle da pressão arterial através de modificações de hábitos de vida ${ }^{(6)}$.

Uma das principais estratégias para prevenção e/ou controle destes fatores é a educação em saúde, que favorece a "desalienação, a transformação e a emancipação" (7: 235) dos indivíduos envolvidos. Porquanto permite ao portador de doença crônica a reflexão e a percepção da saúde como um direito social $^{(7)}$. Assim, ao participar das atividades educativas o usuário, mediante a apreensão de conhecimentos relativos à sua condição, pode ter voz ativa no processo de cuidados e ter opções frente a sua patologia, mediante avaliação das conseqüências de cada regime proposto ${ }^{(8)}$. Para que a opção aconteça é necessário que o usuário conheça todos os aspectos relativos à sua doença, neste sentido, questiona-se: "Qual o conhecimento dos portadores de doença crônica sobre o seu adoecimento e tratamento?"

Diante ao exposto, os objetivos desta pesquisa foram identificar o conhecimento sobre a hipertensão arterial, e mediante os conhecimentos apresentados propor intervenções educativas em grupo.

\section{MATERIAIS E MÉTODOS}

Trata-se de pesquisa de intervenção ${ }^{(9)}$, o qual propõe interferência na realidade estudada a fim de modificá-la, ou seja, não se restringe a explicações e propostas de resolução dos problemas, mas de resolvê-los participativamente. Esta pesquisa integra um projeto de extensão, realizada em uma Unidade Básica de Saúde do município de Colombo, Paraná, de março a novembro de 2009, com 35 portadores de doença crônica cadastrados no Programa de Hipertensão e Diabetes (HIPERDIA), com idade entre 18 e 60 anos. 
A pesquisa foi realizada em duas etapas, na primeira utilizou-se a entrevista semiestruturada, gravada, com 35 usuários, com informações sobre dados biossociais, de conhecimento prévio do tratamento e controle da HAS e das possíveis dúvidas, da qual se obteve os temas a serem trabalhados nos encontros em grupo. As necessidades educativas identificadas foram: processo de adoecimento, aspectos fisiopatológicos da doença e suas complicações, a questão da adesão ao tratamento e aceitação da cronicidade.

Todos os entrevistados foram convidados a participar dos encontros em grupo, mas 27 aceitaram. O convite para estes usuários foi feito por meio de contato telefônico e visita domiciliar, e deste total, 17 compareceram a pelo menos um dos 4 encontros grupais, que consistiram em atividades educativas com duração aproximada de uma hora e meia, com a média de quatro participantes da comunidade e cinco da equipe do projeto de extensão.

A disposição dos encontros consistiu de cinco minutos iniciais para apresentação dos objetivos e apresentação dos novos integrantes, sessenta minutos para a realização das dinâmicas que suscitariam as discussões, sendo estas: bingo, jogo da memória e pega varetas ${ }^{(10)}$, estes são adaptações de jogos de uso comum da população com a teoria acerca de assuntos relacionados a hipertensão e diabetes. E trinta minutos para síntese do tema, avaliação e convivência. No último encontro foi realizada uma avaliação do conhecimento adquirido nestas atividades, através do sorteio de perguntas sobre os temas abordados anteriormente.

Os dados obtidos nos encontros de grupo foram tratados mediante a análise de conteúdo temático. Desta, emergiram duas categorias: "compreensão da doença" e "maneiras de cuidar". No que diz respeito aos aspectos éticos o projeto de pesquisa foi aprovado pelo Comitê de Ética do Setor de Ciências da Saúde da Universidade Federal do Paraná (CAAE n 2424.0.000.091-08), e os usuários foram identificados com a letra $\mathrm{U}$ seguido dos algarismos, de acordo com o discurso durante os encontros.

\section{RESULTADOS E DISCUSSÃO}

Dos 35 portadores de doença crônica entrevistados, 28 são do sexo feminino e 07 do sexo masculino, fato encontrado em outros estudos ${ }^{(11-12)}$, e que é atribuído pela busca freqüente das mulheres ao serviço de saúde ${ }^{(12)}$. As idades variaram de 33 a 58 anos, com uma média de 48,7, e predomínio da faixa etária entre 51 a 60 anos com 20 sujeitos. Estes dados vão ao encontro da literatura, visto que nos hipertensos os níveis tensionais tendem a aumentar nesta faixa etária, pois geralmente está associado a arteriosclerose e a outros fatores de risco como a síndrome metabólica que tende a elevar com a idade, aumentando o risco cardiovascular ${ }^{(5)}$.

Do total de usuários entrevistados 26 são em sua maioria casados, 6 são separados/divorciados, 2 viúvos e 1 solteiro. Em relação à escolaridade, 26 possuem o ensino fundamental incompleto, 6 não são alfabetizados e 2 concluíram o ensino médio. Estudo realizado em Fortaleza, Ceará(13) corrobora com os dados encontrados na questão da escolaridade, pois associa o nível de instrução à hipertensão arterial, visto que estas variáveis são inversamente proporcionais.

Em relação à profissão/ocupação, 23 são do lar, 8 possuem atividade remunerada, e 4 são aposentados ou pensionistas da seguridade social, o que corrobora com a amostra dessa pesquisa que em sua maioria era do sexo feminino. Quanto à renda familiar 20 usuários relataram receber de 1 a 3 salários mínimos por mês, 4 ganham de 3 a 5 salários, 4 com menos de um salário mínimo, 6 afirmaram não possuir renda fixa, e 1 não soube informar. Este achado é significativo, pois a renda limitada pode dificultar a adoção de hábitos de vida saudáveis, além de desestimular a continuidade do tratamento ${ }^{(6)}$.

Com a transcrição dos depoimentos dos usuários nos encontros em grupos foram obtidas duas categorias temáticas: "compreensão da doença" e "maneiras de cuidar" que englobam as percepções dos participantes quando questionados sobre o conhecimento das doenças, os possíveis riscos para desenvolvê-las e maneiras de prevenção. Na primeira categoria as respostas foram relacionadas aos motivos que alteram a pressão arterial e diabetes mellitus, como o estresse, "nervoso", angústia, problemas familiares, solidão, não aceitação da idade e a utilização de drogas lícitas, como nos mostra os trechos a seguir:

[… é o nervoso que ataca minha pressão $[\ldots]$ (U5)

O desemprego também (Uo3)

Eu acho que problemas do dia a dia que colaboram (UO7)

O que leva é a angústia e problemas na família [...]. A pressão alta às vezes dá aquela angústia na gente, comer também e que leva a pessoa a fumar bastante cigarro e beber também (Uo9) 
O "nervoso" e o estresse decorrente das preocupações diárias foram relacionados por todos os participantes como motivos desencadeadores da doença, corroborando com achados em estudos sobre o assunto ${ }^{(11,14)}$, pois os usuários convivem com esses fatores diariamente no trabalho, no lar e nas interações interpessoais. Para tanto, o controle emocional é indispensável na prevenção da hipertensão arterial, visto que há relações de caráter prático entre o estresse emocional e/ou crônico na elevação da pressão $\operatorname{arterial}^{(5)}$.

Nota-se que pelo desconhecimento da etiologia multifatorial da hipertensão, os usuários associam seu desenvolvimento aos aspectos emocionais, o que mostra um reducionismo na compreensão da doença ${ }^{(14)}$. Outros sentimentos como angústia e ansiedade decorrentes dos relacionamentos familiares também surgiram implicitamente, de modo a ocasionar fatores de risco para doenças crônicas como etilismo, tabagismo e alimentação inadequada, e que pode ser constatado no depoimento de Uo9.

O tabagismo, o etilismo e o ato de comer são atividades realizadas como métodos para aliviar a sensação de angústia vivida pelo usuário, ou como meio de compensar as funções de cuidado ${ }^{(15)}$. Contudo, para evitar as complicações, os fatores ambientais modificáveis como tabagismo e etilismo devem ser combatidos, com intuito de reduzir os níveis pressóricos e os riscos cardiovasculares, por meio da adesão ao tratamento farmacológico e não-farmacológico, de modo que possa ocasionar mudanças nos hábitos de vida ${ }^{(5,11,14)}$. Porém, além dos fatores modificáveis, a história familiar também compõe o rol de condições de risco, visto que a hereditariedade interfere no desenvolvimento das doenças crônicas ${ }^{(5,11)}$, e isso pode ser verificado na fala de Uo2.

Vem da família, meu pai teve derrame três vezes (Uo2)

O não apoio familiar e o medo de envelhecer também foram citados pelos usuários U13 e U17 como motivos para alterar a pressão arterial, fato que é evidenciado por estudo anterior, uma vez que o suporte familiar pode auxiliar na adesão dos usuários ao tratamento da doença crônica, assim como as demais relações interpessoais, sendo estes apoios considerados essenciais para se manter uma vida feliz e saudável ${ }^{(16)}$. Em estudo realizado com hipertensos, a sensação de solidão com as perdas familiares, o medo de envelhecer e as modificações corporais encontradas corroboram com os achados do presente estudo(17).

A pessoa que tem medo da velhice, de ficar abandonado pelos filhos, os netos que às vezes abandonam [...] a pessoa fica na cama, porque não está preparado, a gente vai envelhecer [...] têm pessoas que se olham e não se conformam (U13)

A gente sozinho não é alegre [...] a gente fica sozinho e não tem com que conversar $[\ldots]($ U17)

Os relatos de como os usuários gerenciam seu tratamento e minimizam os fatores de risco foram agrupados na categoria "maneiras de cuidar", em que além do tratamento medicamentoso, citaram o não medicamentoso, como a alimentação, chás caseiros e o controle emocional. Isso pode ser constatado nos depoimentos a seguir:

Não, só tomo o remedinho. É dois comprimidos por dia que eu tomo, então eu não sinto nada também [...](U12)

Eu faço chá de folha de chuchu [...] diz que é bom e eu faço (U16)

O uso da medicação como uma forma de se cuidar foi evidente nesta amostra, ao responder as entrevistas, nas discussões em grupo nem todos citaram o tratamento medicamentoso como forma de minimizar os riscos da doença, fato que pode justificar a não adesão ao tratamento ou a aceitação crônica da doença, na qual o remédio não é responsável pela sua cura. Desta maneira, os usuários estabelecem medidas para a melhoria das suas condições de saúde, por meio de práticas populares como o uso de chás, por "oferecer respostas às enfermidades e sofrimentos vividos pelas pessoas em seu cotidiano"(18). Este saber popular está associado a experiências empíricas, fundamentadas nos resultados adquiridos pelo uso desses recursos, que são transmitidos de forma difusa no contexto familiar e social num movimento cíclico ${ }^{(18)}$.

Reafirmando a categoria "compreensão da doença”, os usuários combateram os fatores que acreditam ser a causa da doença como o estresse e a alimentação inadequada, conforme as falas abaixo:

Ah, faço de tudo que eu posso. Tudo o que eu já aprendi aqui, sempre estou fazendo, menos sal, menos gordura, menos fritura [...] Dá pra ver que a minha pressão nem está subindo muito[...] $]($ Uo6) 
Ah, eu estou bem controlada,bem calma [...] antes mesmo eu me estressava muito, agora eu já nem me estresso mais $[\ldots](U 15)$

Percebe-se que as mudanças no estilo de vida ocorreram em função da necessidade de ter que conviver com a doença. Achados semelhantes vão ao encontro desta pesquisa, sendo que alguns usuários mencionaram alteração dos hábitos diários ${ }^{(14)}$. Entretanto, sabe-se que esta adequação aos novos hábitos nem sempre é fácil, como vemos na fala de U08, pois os costumes geralmente estão imbricados nos aspectos sócio-culturais ${ }^{(18)}$.

Uma coisa que eu faço pouco é isso, tomar água (Uos)

No último encontro ocorreu a avaliação das atividades educativas, na qual os pesquisadores levaram perguntas pré-formuladas sobre os assuntos abordados nos encontros e cada participante sorteava e respondia junto com seus colegas. Esta avaliação permitiu uma abertura para a troca de informações, e foi ao encontro do preconizado neste tipo de abordagem, pois ao expressarem suas idéias, possibilitam melhores condições de cuidado de si e dos outros ${ }^{(5)}$.

\section{CONSIDERAÇÕES FINAIS}

As necessidades educativas apontadas pelos sujeitos deste estudo se referem a aspectos fisiopatológicos da doença e suas complicações, do processo de adoecimento, da adesão ao tratamento e aceitação da cronicidade. Nas duas categorias temáticas percebe-se que os usuários portadores de Hipertensão Arterial e Diabetes Mellitus atribuem o desenvolvimento das doenças a partir dos motivos que as alteram, como o estresse, "nervoso", angústia, problemas familiares, solidão, não aceitação da idade e a utilização de drogas lícitas, e para amenizar estes fatores de risco utilizam o tratamento medicamentoso e o não-medicamentoso como a alimentação, chás caseiros e o controle emocional.

Observou-se que os usuários apesar de não saber definir as patologias crônicas aqui apresentadas, conhecem o que é a doença, o seu tratamento, os fatores de risco e as possíveis complicações. Entretanto, entre ter o conhecimento sobre estes assuntos e assumir o tratamento existe uma lacuna, que o presente trabalho não pode responder. As limitações desta pesquisa referem-se ao reduzido número de participantes nos encontros de grupos, os quais foram realizados no período da tarde em dias úteis.

Destarte, a pesquisa mostrou que as atividades educativas realizadas com usuários portadores de doenças crônicas favoreceram a troca de experiências de todos os envolvidos, principalmente por possibilitar a estes a livre expressão de suas idéias, sem censuras e com isto, proporcionar a reflexão e a possibilidade de que estes gerenciem seus tratamentos, e com o apoio dos enfermeiros identifiquem maneiras de cuidar da saúde.

\section{REFERÊNCIAS}

1 Organização Mundial de Saúde. Cuidados inovadores para condições crônicas: componentes estruturais de ação: relatório mundial. Brasília: Ministério da Saúde; 2003.

2 Organização Pan-Americana da Saúde. Prevenção de doenças crônicas: um investimento vital. Brasília: Organização Mundial da Saúde; 2005.

3 Brasil. Agência Nacional de Saúde Suplementar. Promoção da saúde e prevenção de riscos e doenças na saúde suplementar: manual técnico. Rio de Janeiro: ANS; 2007.

4 Brasil. Ministério da Saúde. Informações de Saúde. Morbidade e informações epidemiológicas [Internet]. [citado 2009 nov 01]. Disponível em: www. datasus.gov.br.

5 Sociedade Brasileira de Cardiologia, Sociedade Brasileira de Hipertensão, Sociedade Brasileira de Nefrologia. VI Diretrizes Brasileiras de Hipertensão Arterial ¿Internet]. São Paulo: SBC, SBH, SBN; 2010 [ citado 2010 out 20]. Disponível em http://publicacoes.cardiol.br/ consenso/2010/Diretriz_hipertensao_associados.pdf.

6 Fortes NA, Lopes VO. Análise dos fatores que interferem no controle da pressão arterial de pessoas acompanhadas numa unidade básica de atenção à saúde da família. Texto Contexto Enferm. 2004; 13(1): 26-34.

7 Toledo MM, Rodrigues SC, Chiesa AM. Educação em saúde no enfrentamento da hipertensão arterial: uma nova ótica para um velho problema. Texto Contexto Enferm. 2007 abr-jun; 16(2): 233-8.

8 Mantovani MF, Mendes FRP. A condição crônica de saúde: do diagnóstico a gestão cotidiana da situação. In: Trentini M, Paim L, organizadores. Desafios inerentes às situações crônicas no contex to de saúde. No prelo 2010. 
9 Tobar F, Yalour MR. Como fazer teses em saúde pública: conselhos e idéias para formular projetos e redigir teses e informes de pesquisas. Rio de Janeiro: Fiocruz; 2001.

10 Mantovani MF, Mazza VA, Falcão NS, Gariba IM, Bandeira JM, Rodrigues JAP. La influencia de los juegos educativos en la comprensión y adhesión al tratamiento de la hipertensión arterial. Rev Enferm Herediana. 2008; 1 (2): 130-3.

11 Mantovani MF, Ulbrich EM, Pinotti S, Giacomozzi LM, ZZZ, Labronici LM, Sarquis LMM. O significado e a representação da doença crônica: conhecimento do portador de hipertensão arterial acerca de sua enfermidade. Cogitare Enferm. 2008; 13(3): 336-42.

12 Damasceno MMC, Almeida PC, Almeida VCF, Macedo SF, Silva ARV. Perfil dos níveis pressóricos e glicêmicos de funcionários de instituições públicas hospitalares de Fortaleza-Ceará. Esc Anna Nery. 2006; $10(2)$ : 228-34.

13 Rolim M.Castro M. Compliance To the Hypertension Control Program and the Standardized Nursing Results: an exploratory study. Online Braz J Nurs [Internet]. 2007 apr 21 [cited 2010 mar 17]; 6(1).
Available from: http://www.objnursing.uff.br/index. $\mathrm{php} /$ nursing/article/view/713.

14 Péres DS, Magna JM, Viana LA. Portador de hipertensão arterial: atitudes, crenças, percepções, pensamentos e práticas. Rev Saúde Pública. 2003; 37 (5): 653-642.

15 Lima MT, Bucher JSNF, Lima JWO. Hipertensão Arterial: conhecimentos, atitudes e práticas. Cad. Saúde Pública. 2004; 20(4): 1079-1087.

16 Waidman M.Rocha A.Paschoa A.Radovanovic C. Experiencing problems of the health in family: the implementation of the theoretical-methodological proposal of care. Online Braz J Nurs [Internet]. 2007 jan 20 [cited 2010 march 17]; 6(0). Available from: http://www.objnursing.uff.br/index.php/ nursing/article/view/648.

17 Baldissera VDA, Carvalho MDB, Pelloso SM. Adesão ao tratamento não-farmacológico entre hipertensos de um centro de saúde escola. Rev Gaúcha Enferm. 2009; 30 (1): 27-32.

18 Siqueira KM, Barbosa MA, Brasil VV, Oliveira LMC, Andraus LMS. Crenças populares referentes à saúde: apropriação de saberes sócio-culturais. Texto Contexto Enferm. 2006; 15(1): 68-73.

\author{
Endereço do autor / Dirección del autor / \\ Author's address: \\ Elis Martins Ulbrich \\ Av. Lothário Meissner, 632, 3 andar, Jardim Botânico \\ 80210-170, Curitiba, PR \\ E-mail: lilaulbrich@yahoo.com.br
}

